SCIENTIFIC LETTER

\title{
Increase of out-of-hospital cardiac arrests in the male population of the French speaking provinces of Switzerland during the 1998 FIFA World Cup
}

\author{
E Katz, J-T Metzger, J Schlaepfer, M Fromer, D Fishman, L Mayer, M Niquille, L Kappenberger
}

Heart 2005;91:1096-1097. doi: 10.1136/hrt.2004.045195

\begin{abstract}
A cute mental stress and anger are well known triggers of myocardial infarction and sudden cardiac death. It has been described in environmental disasters, such as earthquakes, or during military conflicts. ${ }^{1}$ The emotional intensity of sporting events and the behaviours associated with watching these events, such as smoking, binge drinking, and medical non-compliance, have also been shown to increase the rate of myocardial infarction and sudden cardiac death. $^{2}$ However, recent observations showed a significant decrease in cardiovascular mortality in French men during the 1998 FIFA World Cup which was held in, and won by, France. This decrease was explained by a reduction of mental stress caused by the euphoria of victory. ${ }^{3}$ This competition was also an outstanding sporting event for neighbouring countries; according to FIFA data the total television audience of the 1998 World Cup was estimated at close to 10 million people in Switzerland. Taking into account the similarities in geographic situation, behaviour, and diet among populations of France and French speaking provinces of Switzerland (most of whom supported the French soccer team after the failure of the Swiss team to qualify for the final part of the competition), we were interested to know whether a decrease in out-of-hospital cardiac arrests (OHCA) would also be registered in Switzerland during this event.
\end{abstract}

\section{METHODS}

The MICU (mobile intensive care unit) of the Swiss Emergency Cardiac Care system is qualified both for resuscitation of OHCA and to certify death, and is implicated in the quasi totality of the events. We retrospectively analysed MICU registers in the French speaking provinces of Switzerland (Geneva, Vaud, Fribourg, Neuchâtel), a population of 1.5 million inhabitants, during the 1998 FIFA World Cup (period B, 10 June 1998 to 12 July 1998) and during periods of the same duration preceding (period A, 8 May 1998 to 9 June 1998) and following (period C, 13 July 1998 to 14 August 1998) this competition. Only adult non-traumatic cardiac arrests of presumed cardiac origin were selected for our study. Statistics were calculated using Fisher's exact test.

\section{RESULTS}

The main results of our study are presented in table 1. It was found that $40 \%$ fewer OHCA were registered during the period preceding the FIFA 1998 World Cup $(p=0.044)$ in comparison with the competition period; it was followed by a $60 \%(p=0.001)$ decrease of OHCA after the competition. There was no significant variation in the number of cardiac arrests in women (respectively seven, eight, and six patients suffered from OHCA before, during, and after the competition; $\mathrm{p}=0.96)$. However among males, $46 \%$ fewer OHCA $(\mathrm{p}=0.001)$ were registered during the period preceding the FIFA World Cup, in comparison with the period of
Table 1 Out-of-hospital cardiac arrest (OHCA) in French speaking provinces of Switzerland before, during, and after the 1998 FIFA World Cup, and during the same time intervals in 1997 and in 1999

\begin{tabular}{|c|c|c|c|c|}
\hline & Period A & $\begin{array}{l}\text { Period B } \\
\text { (World Cup) }\end{array}$ & Period C & p Value \\
\hline Total OHCA in 1998 & 27 & 45 & 18 & 0.002 \\
\hline Males & 20 & 37 & 12 & 0.001 \\
\hline Females & 7 & 8 & 6 & NS \\
\hline Mean (SD) age (years) & $66(15)$ & $62(14)$ & $58(15)$ & NS \\
\hline Site of OHCA & & & & \\
\hline Home ( $\%$ of total) & 67 & 64 & 67 & NS \\
\hline $\begin{array}{l}\text { Public place (\% of total) } \\
\text { First registered rhythm }\end{array}$ & 33 & 36 & 33 & NS \\
\hline $\begin{array}{l}\text { Asystole and PEA } \\
\text { (\% of total) }\end{array}$ & 78 & 76 & 78 & NS \\
\hline VF (\% of total) & 22 & 24 & 22 & NS \\
\hline Total OHCA in 1997 & 24 & 20 & 22 & NS \\
\hline Total OHCA in 1999 & 18 & 21 & 22 & NS \\
\hline
\end{tabular}

competition, and a $68 \%(\mathrm{p}=0.001)$ reduction of OHCA was noted during the period following the FIFA World Cup. Despite the fact that call to arrival intervals were short, only one fifth of patients were found in shockable rhythms. The OHCA long term survival rate was 6\% in 1998.

\section{DISCUSSION}

In our study, an increase of OHCA among the male population of the French speaking provinces of Switzerland during the FIFA 1998 World Cup was noted. Recent observations by Berthier and colleagues, based on analysis of death certificates (recorded in French death statistics system), showed a decrease in cardiovascular mortality in French men the day that France won the 1998 FIFA World Cup. This decrease was attributed to a reduction in stress because of the euphoria of victory. ${ }^{3}$ This study and a study completed by Toubiana and colleagues did not find an increase in French cardiovascular mortality during the 1996 European football championship; this difference may be explained by different methods of observation, because of the limited value of death certificates in the diagnosis of OHCA as previously reported..$^{45}$ The similarities in geographical situation, behaviour, and diet between the populations of the French speaking provinces of Switzerland and France led us to suppose that our results could be also obtained in France if

Abbreviations: AED, automated external defibrillator; FIFA, Fédération Internationale de Football Association; MICU, mobile intensive care unit; OHCA, out-of-hospital cardiac arrest 
our study were replicated using MICU data. Although we cannot prove that all patients of our study who suffered from OHCA were football supporters, the increase in mental stress can contribute to an increase in sympathetic nervous system stimulation and circulating catecholamines, and induce a state of hypercoagulability. In addition, increased alcohol and tobacco consumption, a decrease in physical activity, and medical non-compliance during major sporting events, such as the FIFA World Cup, could contribute to an increase in the rate of sudden cardiac deaths among the male population of the French speaking provinces of our country. On the basis of our observations and of previous studies which focused on the impact of soccer competitions on cardiovascular mortality, we recommend that general practitioners inform their patients, and members of their families, of the risk of myocardial infarction and sudden cardiac death, and to observe medical compliance during major sporting events. The reinforcement of the emergency cardiac care system (increased number of emergency phone operators, physicians and paramedics on call) before major sporting events could also be suggested. The low incidence of shockable rhythms, despite short call to arrival intervals, should also be mentioned. In light of this fact, programmes which improve emergency communication systems and lower the threshold for calls to first responder must be implemented. More information about practical measures to adopt in case of chest pain or cardiac arrest must be provided to the general public via the media and physicians before major sporting events. The changes in AED deployment strategies and development of public access defibrillation are also expected to reduce the incidence of sudden cardiac death.

\section{ACKNOWLEDGEMENTS}

We thank Professor Jiri Dvorak, FIFA Medical Committee, Zurich, Switzerland, for carefully reviewing the article.

\section{Authors' affiliations}

E Katz, J-T Metzger, J Schlaepfer, M Fromer, L Kappenberger, Department of Cardiology, Centre Hospitalier Universitaire Vaudois, Lausanne, Switzerland

D Fishman, Emergency Department, Centre Hospitalier Universitaire Vaudois

L Mayer, Anesthesiology Department, Hôpital Cantonal, Fribourg, Switzerland

M Niquille, Emergency Department, Hôpital Cantonal Universitaire, Genève, Switzerland

Correspondence to: Eugène Katz, MD, Department of Cardiology, Centre Hospitalier Universitaire Vaudois, 1011, Lausanne, Switzerland; Eugene.Katz@hospvd.ch

Accepted 11 October 2004

\section{REFERENCES}

1 Stalnikowicz R, Tsafrir A. Acute psychosocial stress and cardiovascular events. Am J Emerg Med 2002;20:488-91.

2 Caroll D, Ebrahim S, Tilling K, et al. Admissions for myocardial infarction and World Cup football: database survey. BMJ 2002;325:1439-42.

3 Berthier F, Boulay F. Lower myocardial infarction mortality in French men the day France won the 1998 World Cup of football. Heart 2003;89:555-6.

4 Toubiana L, Hanslik T, Letrillart L. French cardiovascular mortality did not increase during 1996 European football championship. BMJ 2001;322:1306.

5 Iribarren C, Crow RS, Hannan PJ, et al. Validation of death certificate diagnosis of out-of-hospital sudden cardiac death. Am J Cardiol 1998:82:50-3.

\section{IMAGES IN CARDIOLOGY}

\section{Histology of a surgically removed stenotic modified Blalock-Taussig shunt after previous endovascular stenting}

$\mathrm{T}$

he histological image of a surgically removed stenotic $3.5 \mathrm{~mm}$ modified polytetrafluoroethylene (PTFE)

Blalock-Taussig shunt is shown here. The shunt had been implanted in a 7 week old infant with double outlet left ventricle, transposition of the great arteries, ventricular septal defect, and severe pulmonary stenosis. Eleven months later a coronary stent (MAC $17 \times 4 \mathrm{~mm}$, AMG International GmbH, Raesfeld-Erle, Germany) was successfully implanted into the shunt because of progressive shunt narrowing. After a period of one year with the infant in a stable condition, a gradual decline of oxygen saturation was noted. Follow up angiography revealed significant narrowing at the pulmonary end of the stented shunt. Subsequently, the shunt was removed during corrective surgery at the age of 31 months. The excised tissue specimen was fixed in formalin and embedded in hydroxyethylmethacrylate (Technovit 7200, Kulzer \& Co, Wehrheim, Germany). Sections were obtained by sectioning of the resin block using a diamond cutter and subsequent grinding. Histology demonstrates significant narrowing of the lumen. The stent struts are neighbouring the PTFE material indicating effective stenting at the time of implantation 18 months before explantation. The bulk of obstruction is caused by neointima formation. Histology reveals the contribution of thrombus apposition to lumen narrowing in addition to neointima hyperplasia. Further neointimal growth at the lumen sided surface of the thrombus indicates a time interval of several weeks between thrombus formation and explantation.

\footnotetext{
M Sigler

D Bartmus

T Paul

msigler@gwdg.de
}

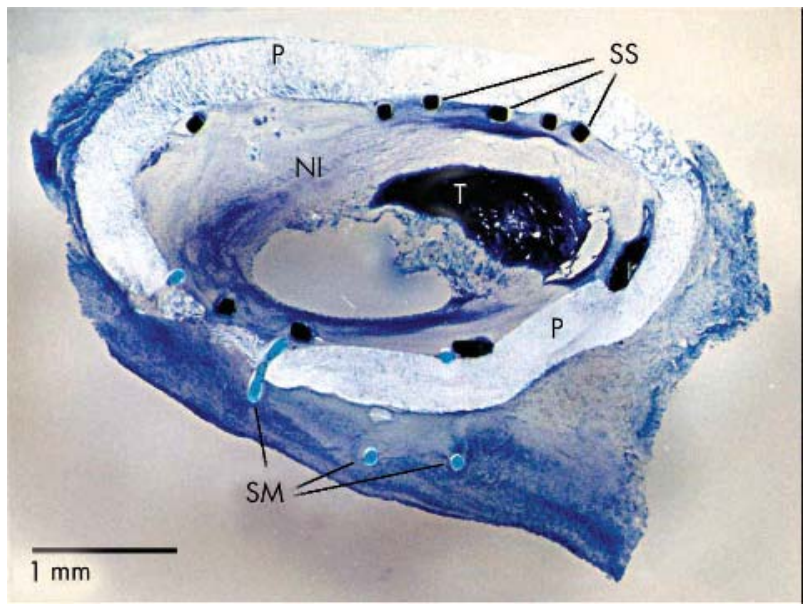

Ground section of the pulmonary end of a stented modified BlalockTaussig shunt showing significant narrowing of the lumen. Richardson blue staining. $\mathrm{NI}$, neointima; P, PTFE membrane; SM, suture material; SS, stent strut; $T$, thrombus material. 